\title{
Assessing the influence of metallicity on fragmentation of proto-galactic gas
}

\author{
Anne-Katharina Jappsen ${ }^{1,2}$, Simon C. O. Glover ${ }^{2}$, Ralf S. Klessen ${ }^{3}$ \\ and Mordecai-Mark Mac Low ${ }^{4}$ \\ ${ }^{1}$ Canadian Institute for Theoretical Astrophysics, University of Toronto, \\ Toronto, ON M5S 3H8, Canada \\ email: jappsen@cita.utoronto.ca \\ ${ }_{2}^{2}$ Astrophysikalisches Institut Potsdam, An der Sternwarte 16, D-14482 Potsdam, Germany \\ email: sglover@aip.de \\ ${ }^{3}$ Insitut für Theoretische Astrophysik/Zentrum für Astronomie der Universität Heidelberg, \\ Albert-Überle-Str. 2, D-69120, Germany \\ email: rklessen@ita.uni-heidelberg.de \\ ${ }^{4}$ Department of Astrophysics, American Museum of Natural History, \\ 79th Street at Central Park West, New York, NY 10024-5192, USA \\ email: mordecai@amnh.org
}

\begin{abstract}
In cold dark matter cosmological models, the first stars to form are believed to do so within small protogalaxies. We study the influence of low levels of metal enrichment on the cooling and collapse of ionized gas in these protogalactic halos using three-dimensional, smoothed particle hydrodynamics simulations.
\end{abstract}

Keywords. stars: formation, methods: numerical, hydrodynamics, early Universe

We wish to understand how the evolution of early protogalaxies changes once the gas forming them has been enriched with small quantities of heavy elements, which are produced and dispersed into the intergalactic medium by the first supernovae. Adding heavy elements to the gas increases its ability to radiate heat and to control its temperature. It has been argued that enrichment beyond a certain 'critical metallicity' allows the first solar-mass stars to form, while protogalaxies with fewer metals form only massive stars, with masses greater than a hundred times solar. This idea has been accepted as a working hypothesis by many cosmologists, but it has yet to be rigorously tested. Although observational tests will not be feasible until the next generation of telescopes become available, we can begin to test this idea numerically, using high-resolution hydrodynamic simulations that incorporate the effects of the appropriate chemical and thermal processes. Our initial conditions represent protogalaxies forming within a fossil $\mathrm{H}$ II region a previously ionized region that has not yet had time to cool and recombine. We vary the initial redshift between $z=15$ and $z=30$ and the dark matter halo masses between $5 \times 10^{4}$ and $10^{7} \mathrm{M}_{\odot}$. The gas mass resolution lies between 20 and $400 \mathrm{M}_{\odot}$ (Jappsen et al. 2007). Our simulations demonstrate that for metallicities $Z \leqslant 10^{-3} \mathrm{Z}_{\odot}$, metal line cooling alters the density and temperature evolution of the gas by less than $1 \%$ compared to the metal-free case at low densities $\left(n<1 \mathrm{~cm}^{-3}\right)$ and high temperatures $(T>2000 \mathrm{~K})$. We also present the results of high-resolution simulations using particle splitting (Kitsionas \& Whitworth 2002) to improve resolution in regions of interest.

\section{References}

Kitsionas, S., \& Whitworth, A. P. 2002, MNRAS, 330, 129

Jappsen, A.-K., Glover, S. C. O., Klessen, R. S., \& Mac Low, M.-M. 2007, ApJ, 660, 1332 